\title{
ЛИНЕЙНЫЕ И КВАДРАТИЧНЫЕ НЕЧЕТКИЕ ИЕРАРХИЧЕСКИЕ ОКРЕСТНОСТНЫЕ МОДЕЛИ ПРОЦЕССА ПРОИЗВОДСТВА ХОЛОДНОКАТАНОЙ СТАЛИ
}

\author{
И. А. Седых \\ Липецкий государственньй технический университет
}

Поступила в редакцию 05.03.2019 г.

\begin{abstract}
Аннотация. Разработка имитационной модели процесса производства холоднокатаной стали является актуальной, так как позволяет выполнять прогнозирование механических свойств, формирующихся после отжига, без проведения натурного эксперимента. Целью работы является применение окрестностного подхода к моделированию рассматриваемого процесса на основе линейных и квадратичных динамических окрестностных моделей, проведение сравнения результатов моделирования с производственными данными. Приведено определение динамических окрестностных моделей с нечеткой иерархической структурой, отличающихся нечеткими двухуровневыми окрестностными связями между узлами первого и второго уровня модели. Построены линейные и квадратичные четкие неиерархические и нечеткие иерархические динамические окрестностные модели для расчёта предела текучести, временного сопротивления и относительного удлинения. Выполнен анализ модельных и производственных данных на основе относительных ошибок идентификации и моделирования для рассмотренных моделей.

Ключевые слова: динамические окрестностные модели, линейные и квадратичные модели, нечеткая иерархическая структура, процесс производства холоднокатаной стали, механические свойства металла.

Annotation. The development of a simulation model for the production process of cold-rolled steel is relevant, as it allows the prediction of mechanical properties that are formed after annealing, without conducting a full-scale experiment. The aim of the work is to apply a neighborhood approach to modeling the process under consideration based on linear and quadratic dynamic neighborhood models, and to compare the simulation results with production data. The definition of dynamic neighborhood models with a fuzzy hierarchical structure, distinguished by fuzzy two-level neighborhood connections between nodes of the first and second level of the model is given. Constructed linear and quadratic clear non-hierarchical and fuzzy hierarchical dynamic neighborhood models to calculate the yield strength, temporal resistance and relative elongation. The analysis of model and production data is performed on the basis of relative identification and modeling errors for the considered models.
\end{abstract}

Keywords: dynamic neighborhood models, linear and quadratic models, fuzzy hierarchical structure, the production process of cold-rolled steel, the mechanical properties of the metal.

\section{ВВЕДЕНИЕ}

При производстве холоднокатаной стали в процессе непрерывного отжига важным является определение, контроль и управление такими механическими свойствами металла как предел текучести, временное сопротивление, относительное удлинение.

(с) Седых И. А., 2019
Пластичность - это свойство твердых тел сохранять часть деформации при снятии нагрузок, которые их вызвали. Предел текучести - механическая характеристика материала, характеризующая напряжение, при котором деформации продолжают расти без увеличения нагрузки. Предел текучести характеризует способность пластичного материала к деформации без происхождения в нем необратимых изменений. 


\section{И. А. Седblx}

Временное сопротивление или предел прочности - максимальное механическое напряжение, при превышении которого происходит разрушение материала, подвергаемого деформации. Временное сопротивление для металлов определяется при разрыве цилиндрического образца, предварительно подвергнутого отжигу.

Показателем пластичности является относительное удлинение, которое определяется при тех же испытаниях, что и временное сопротивление. Относительное удлинение отношение приращения длины образца после его разрыва к первоначальной расчетной длине [1-2].

Разработка имитационной модели процесса производства холоднокатаной стали является актуальной на сегодняшний день, так как позволяет выполнять прогнозирование механических свойств стали, формирующихся в процессе непрерывного отжига без проведения натурного эксперимента.

В [3] разработаны статические линейные регрессионные модели зависимости механических свойств стали только от химического состава, а в [4] - и от других технологических параметров производства: температурных режимов горячей прокатки и суммарных обжатий при холодной прокатке.

Однако производство холоднокатаной стали является пространственно-распределенным динамическим процессом, поэтому важной является разработка имитационной модели, отражающей динамику рассматриваемого процесса и позволяющей контролировать результаты на промежуточных этапах производства. Удобным инструментом для моделирования таких сложных распределенных производственных процессов и систем являются окрестностные модели [5-8], основные понятия которых рассмотрены в [9-10].

Целью данной работы является применение окрестностного подхода к моделированию процесса производства холоднокатаной стали [11] на основе линейных и квадратичных динамических окрестностных моделей с нечеткой иерархической структурой, а также неиерархических окрестностных моделей, проведение сравнения результатов модели- рования с производственными данными. Следует отметить, что в отличие от [3-4], где рассматриваются модели одной конкретной марки стали, необходимо разработать имитационную модель для всего обрабатываемого сортамента.

\section{1. МАТЕРИАЛЫ И МЕТОДЫ}

\section{1. Определение нечеткой иерархической окрестностной модели}

Динамическая окрестностная модель [1112] с нечеткой иерархической структурой (далее - нечеткая иерархическая окрестностная модель) задается набором $N S_{\text {IER }}=(\tilde{N}, X, V, Z, G, X[0])$, где:

1. $\tilde{N}=\left(A, O_{x}, O_{v}, \tilde{O}_{i e r}\right)-$ двухуровневая нечеткая структура модели, состоящая из множества узлов первого уровня $A=\left\{a_{1}, a_{2}, \ldots, a_{n}\right\}$, окрестностей узлов по состояниям $O_{x}$, окрестностей узлов по управляющим воздействиям $O_{v}$, нечетких иерархических окрестностных связей между узлами первого и второго уровня $\tilde{O}_{\text {ier }}$.

Каждому узлу первого уровня $a_{i} \in A$ поставлено в соответствие нечеткое множество узлов второго уровня $\tilde{O}_{i e r}[i]=\left\{a_{i}^{1}, \ldots, a_{i}^{c}\right\}$, каждый из которых является окрестностной моделью. При этом $a_{i}^{b} \in \tilde{O}_{i e r}[i], b=1, \ldots, c$ с некоторой функцией принадлежности $W_{i}^{b}: X_{O_{x}\left[a_{i}\right]} \times V_{O_{v}\left[a_{i}\right]} \rightarrow \mathbf{R}_{[0,1]}$.

Для всех узлов второго уровня $a_{i}^{b}$ $O_{x}\left[i^{b}\right]=O_{x}[i] ; O_{v}\left[i^{b}\right]=O_{v}[i]$.

2. $X \in R^{\sum_{i=1}^{n} p_{i}}-$ блочный вектор состояний модели в текущий момент времени.

3. $V \in R^{\sum_{i=1}^{n} m_{i}}-$ блочный вектор управляющих воздействий в текущий момент времени.

4. $Z \in \mathbf{R}_{+}^{n \times n}$ - матрица временных задержек сигналов в узлах.

5. $G: X_{O_{x}} \times V_{O_{v}} \rightarrow X-$ функция пересчета состояний окрестностной модели, где $X_{O_{x}}-$ множество состояний узлов, входящих в окрестность $O_{x} ; V_{O}$ - множество управлений узлов, входящих в окрестность $O_{v}$.

6. $X[0]$ - начальное состояние модели. 


\section{2. Линейная и квадратичная нечеткие иерархические окрестностнье модели}

В линейном случае для узла второго уровня $a_{i}^{b} \in \tilde{O}_{i e r}[i]$ функция пересчета состояний $G_{i}^{b}$ имеет вид:

$$
\begin{gathered}
X\left[t+1, i^{b}\right]=G_{i}^{b}[t]=g_{c}\left[i^{b}\right]+ \\
+\sum_{a_{j} \in O_{x}\left[i^{b}\right]} g_{x}\left[i^{b}, j\right] X[t, j]+\sum_{a_{k} \in O_{v}\left[i^{b}\right]} g_{v}\left[i^{b}, k\right] V[t, k],
\end{gathered}
$$

где $a_{j}, a_{k} \in A \quad(j, k=1, \ldots, n)$ - узлы первого уровня $\quad$ модели; $g_{x}\left[i^{b}, j\right] \in R^{p_{i} \times p_{j}}$, $g_{v}\left[i^{b}, k\right] \in R^{p_{i} \times m_{k}}, \quad g_{c}\left[i^{b}\right] \in R^{p_{i} \times 1}-$ матрицы-параметры.

Квадратичная функция $G_{i}^{b}$ задается равенством:

$$
\begin{gathered}
X\left[t+1, i^{b}\right]=G_{i}^{b}[t]=g_{c}\left[i^{b}\right]+ \\
+\sum_{a_{j} \in O_{x}\left[i^{b}\right]} g_{x}\left[i^{b}, j\right] \cdot X[t, j]+ \\
+\sum_{a_{k} \in O_{v}\left[i^{b}\right]} g_{v}\left[i^{b}, k\right] \cdot V[t, k]+ \\
+\sum_{a_{j} \in O_{x}\left[i^{b}\right]} \sum_{a_{k} \in O_{v}\left[i^{b}\right]} g_{x v}\left[i^{b}, j, k\right] \bullet X[t, j] \cdot V[t, k]+ \\
+\sum_{a_{j} \in O_{x}\left[i^{b}\right]} \sum_{a_{k} \in O_{x}\left[i^{b}\right]} g_{x x}\left[i^{b}, j, k\right] \bullet X[t, j] \cdot X[t, k]+ \\
+\sum_{a_{j} \in O_{v}\left[i^{b}\right]} \sum_{a_{k} \in O_{v}\left[i^{b}\right]} g_{v v}\left[i^{b}, j, k\right] \bullet V[t, j] \cdot V[t, k],
\end{gathered}
$$

где $g_{x v}\left[i^{b}, j, k\right] \in R^{p_{j} \times p_{i} \times m_{k}}, g_{x x}\left[i^{b}, j, k\right] \in R^{p_{j} \times p_{i} \times p_{k}}$, $g_{v v}\left[i^{b}, j, k\right] \in R^{m_{j} \times p_{i} \times m_{k}}$ - блочные матрицы-параметры.

Произведение $g_{x v}\left[i^{b}, j\right] \bullet X[t, j]$ в (2) является блочным и выполняется по следующему правилу:

$$
\begin{gathered}
g_{x v}\left[i^{b}, j, k\right] \bullet X[t, j]= \\
=\sum_{d=1}^{p_{j}} g_{x v}\left[d, i^{b}, j, k\right] \cdot x[t, j, d],
\end{gathered}
$$

где $g_{x v}\left[d, i^{b}, j, k\right] \in R^{p_{i} \times m_{k}}-d$-й элемент блочной матрицы $g_{x v}\left[i^{b}, j, k\right], x[t, j, d] \in R-d$-й элемент вектора $X[t, j], d=1, \ldots, p_{j}$. Аналогично определяются и произведения $g_{x x}\left[i^{b}, j, k\right] \bullet X[t, j], g_{v v}\left[i^{b}, j, k\right] \bullet V[t, j]$.

Для каждого узла первого уровня $a_{i} \in A$ функция $G_{i}$ будет иметь вид:

$$
X[t+1, i]=G_{i}[t]=\frac{\sum_{b=1}^{c} W_{i}^{b}[t] \cdot G_{i}^{b}[t]}{\sum_{b=1}^{c} W_{i}^{b}[t]},
$$

где $W_{i}^{b}[t]$ - степень принадлежности узла второго уровня $a_{i}^{b}$ узлу первого уровня $a_{i}$ в момент времени $t$.

Идентификация $[11,13]$ нечеткой иерархической окрестностной модели заключается в нахождении функций принадлежности $W_{i}^{b}$ на основе нечеткой кластеризации [14-15] и параметров функций пересчета состояний $G_{i}^{b}$ для всех узлов второго уровня $a_{i}^{b}$ $(i=1, \ldots, n ; b=1, \ldots, c)$.

Относительная ошибка идентификации модели вычисляется по формуле:

$\delta=\frac{100 \%}{N \cdot n} \sum_{i=1}^{n}\left(\frac{1}{p_{i}} \sum_{d=1}^{p_{i}} \sum_{j=1}^{N}\left|\frac{x_{j}[t, i, d]-\hat{x}_{j}[t, i, d]}{\max _{j} x_{j}[t, i, d]}\right|\right)$,

где $N$ - объем выборки; $x_{j}[t, i, d]-d$-й элемент вектора состояний $i$-го узла $j$-й строки данных в выборке; $\hat{x}_{j}[t, i, d]-d$-й элемент $i$-го узла $j$-й строки данных вектора состояний, посчитанного по модели.

\section{2. РЕЗУЛЬТАТЫ И ИХ ОБСУЖДЕНИЕ}

\section{1. Окрестностные модели процесса производства холоднокатаной стали}

Обработка холоднокатаной стали подразумевает несколько этапов. На рассмотренные выше механические свойства, а именно предел текучести, временное сопротивление и относительное удлинение, наибольшее влияние оказывают выплавка стали в конвертере, горячая прокатка и непрерывный отжиг после холодной прокатки. Рассмотрим граф структуры окрестностной модели процесса производства холоднокатаной стали (рис. 1) [11].

На рис. 1 обозначены следующие узлы: $a_{1}$ - конвертер, $a_{2}$ - стан горячей прокатки, $a_{3}$ - агрегат непрерывного отжига.

Следовательно, окрестностные связи между узлами окрестностной модели равны: $O_{x}[1]=\{\varnothing\}, \quad O_{x}[2]=\left\{a_{1}\right\}, \quad O_{x}[3]=\left\{a_{1}, a_{2}\right\}$, $O_{v}[i]=\left\{a_{i}\right\}, \quad i=1,2,3$. При этом $X[t, 1] \in R^{12}$, $X[t, 2] \in R^{3}, \quad X[t, 3] \in R^{3}, \quad V[t, 1] \in R^{12}$, $V[t, 2] \in R^{2}, V[t, 3] \in R^{4}$.

Узлы окрестностной модели соответствуют цехам производства холоднокатаной ста- 


\section{И. А. Седых}

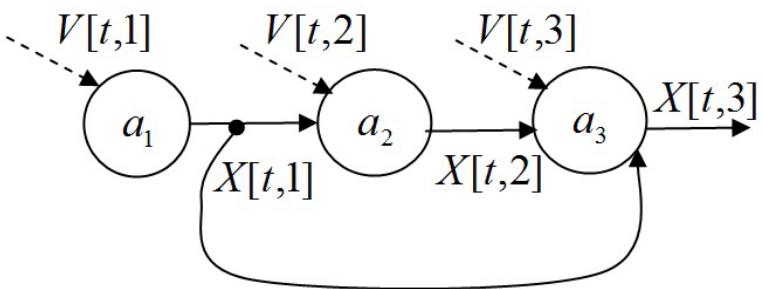

Рис.1. Граф структуры окрестностной модели проиесса производства холоднокатаной стали

ли, а управляющие воздействия и состояния входным факторам и внутренним состояниям соответствующих узлов. Управляющими воздействиями в узлах модели являются: химический состав, скоростные режимы горячей прокатки, температурные и скоростные режимы непрерывного отжига; состояниями - температурные режимы горячей прокатки, механические свойства, формирующиеся в процессе непрерывного отжига. Узел $a_{3}$ является выходным узлом системы. В узле $a_{3}$ состояние $X[t, 3]=(x[t, 3,1] \quad x[t, 3,2] \quad x[t, 3,3])^{T}$, где $x[t, 3,1]$ - предел текучести, $x[t, 3,1]$ - временное сопротивление, $x[t, 3,3]-$ относительное удлинение в текущий момент времени.

Пусть $\tilde{O}_{i e r}[i]=\left\{a_{i}^{1}, \ldots, a_{i}^{c}\right\}$. Тогда для каждого узла второго уровня $a_{i}^{b} \in \tilde{O}_{i e r}[i]$ функция пересчета состояний $G_{i}^{b}$ :

$$
\left\{\begin{array}{l}
X\left[t+1,1^{b}\right]=G_{1}^{b}(V[t, 1]) ; \\
X\left[t+1,2^{b}\right]=G_{2}^{b}(X[t, 1], V[t, 2]) ; \\
X\left[t+1,3^{b}\right]=G_{3}^{b}(X[t, 1], X[t, 2], V[t, 3]),
\end{array}\right.
$$

где $X\left[t+1, i^{b}\right]$ - вектор состояний $b$-го подузла $i$-го узла первого уровня в момент времени $t+1 ; V[t, i]$ и $X[t, i]$ - векторы управляющих воздействий и состояний $i$-го узла первого уровня в момент времени $t$ соответственно.

В линейном случае система (6) имеет вид:

$$
\left\{\begin{array}{l}
X\left[t+1,1^{b}\right]=g_{c}\left[1^{b}\right]+g_{v}\left[1^{b}, 1\right] V[t, 1] ; \\
X\left[t+1,2^{b}\right]=g_{c}\left[2^{b}\right]+g_{x}\left[2^{b}, 1\right] X[t, 1]+ \\
+g_{v}\left[2^{b}, 2\right] V[t, 2] ; \\
X\left[t+1,3^{b}\right]=g_{c}\left[3^{b}\right]+g_{x}\left[3^{b}, 1\right] X[t, 1]+ \\
+g_{x}\left[3^{b}, 2\right] X[t, 2]+g_{v}\left[3^{b}, 3\right] V[t, 3],
\end{array}\right.
$$

где $g_{c}\left[1^{b}\right] \in R^{12 \times 1}, g_{c}\left[2^{b}\right] \in R^{3 \times 1}, g_{c}\left[3^{b}\right] \in R^{3 \times 1}-$ матрицы свободных членов модели; $g_{x}\left[2^{b}, 1\right] \in R^{3 \times 12}, g_{x}\left[3^{b}, 1\right] \in R^{3 \times 12}, g_{x}\left[3^{b}, 3\right] \in R^{3 \times 4}-$ матрицы коэффициентов перед векторами состояний; $\quad g_{v}\left[1^{b}, 1\right] \in R^{12 \times 12}, \quad g_{v}\left[2^{b}, 2\right] \in R^{3 \times 2}$, $g_{v}\left[3^{b}, 3\right] \in R^{3 \times 4}-$ матрицы коэффициентов перед векторами управляющих воздействий.

В случае квадратичных функций пересчета состояний система (6) имеет вид:

$$
\left\{\begin{array}{l}
X\left[t+1,1^{b}\right]=g_{c}\left[1^{b}\right]+g_{v}\left[1^{b}, 1\right] V[t, 1]+ \\
+g_{v v}\left[1^{b}, 1,1\right] \bullet V[t, 1] \cdot V[t, 1] \\
X\left[t+1,2^{b}\right]=g_{c}\left[2^{b}\right]+g_{x}\left[2^{b}, 1\right] X[t, 1]+ \\
+g_{v}\left[2^{b}, 2\right] V[t, 2]+g_{x v}\left[2^{b}, 1,2\right] \bullet X[t, 1] \cdot V[t, 2]+ \\
+g_{x x}\left[2^{b}, 1,1\right] \bullet X[t, 1] \cdot X[t, 1]+ \\
+g_{v v}\left[2^{b}, 2,2\right] \bullet V[t, 2] \cdot V[t, 2] \\
X\left[t+1,3^{b}\right]=g_{c}\left[3^{b}\right]+g_{x}\left[3^{b}, 1\right] X[t, 1]+ \\
+g_{x}\left[3^{b}, 2\right] X[t, 2]+g_{v}\left[3^{b}, 3\right] V[t, 3]+ \\
+g_{x v}\left[3^{b}, 1,3\right] \bullet X[t, 1] \cdot V[t, 3]+ \\
+g_{x v}\left[3^{b}, 2,3\right] \bullet X[t, 2] \cdot V[t, 3]+ \\
+g_{x x}\left[3^{b}, 1,1\right] \bullet X[t, 1] \cdot X[t, 1]+ \\
+g_{x x}\left[3^{b}, 1,2\right] \bullet X[t, 1] \cdot X[t, 2]+ \\
+g_{x x}\left[3^{b}, 2,2\right] \bullet X[t, 2] \cdot X[t, 2]+ \\
+g_{v v}\left[3^{b}, 3,3\right] \bullet V[t, 3] \cdot V[t, 3]
\end{array}\right.
$$

где $g_{x v}\left[2^{b}, 1,2\right] \in R^{12 \times 3 \times 2}, \quad g_{x v}\left[3^{b}, 1,3\right] \in R^{12 \times 3 \times 4}$, $g_{x v}\left[3^{b}, 2,3\right] \in R^{4 \times 3 \times 4}$, $g_{x x}\left[3^{b}, 1,1\right] \in R^{12 \times 3 \times 12}$, $g_{x x}\left[3^{b}, 2,2\right] \in R^{4 \times 3 \times 3}$, $g_{v y}\left[1^{b}, 1,1\right] \in R^{12 \times 12 \times 12}$ $g_{v v}\left[2^{b}, 2,2\right] \in R^{2 \times 3 \times 2}, g_{v v}\left[3^{b}, 3,3\right] \in R^{3 \times 3 \times 3}-$ трехмерные матрицы коэффициентов в произведении, раскрываемом по формуле (3).

\section{2. Сравнение рассмотренных моделей}

Данные для моделирования процесса производства холоднокатаной стали получены на металлургическом производстве и представлены средними для каждой единицы продукции значениями непрерывно измеряемых параметров обработки стали на агрегатах, а также результатами лабораторных анализов отобранных проб металла. Для контроля сформирована равномерная случайная выборка, состоящая из 10 \% исходной. Оставшаяся часть исходной выборки использовалась для идентификации модели. Объемы обучающей и контрольной выборок составляют соответственно 4698 и 521 строку. 
После проведения идентификации систем (4), (7) и (8), то есть нахождения функций принадлежности узлов второго уровня и матриц-параметров, получены результаты, представленные в табл. 1. Для сравнения в табл. 1 также приведены результаты идентификации линейной и квадратичной четких неиерархических окрестностных моделей. Относительная ошибка идентификации находилась по формуле (5).

Таблица 1

Относительная ошибка идентификации, \%

\begin{tabular}{|c|c|c|}
\hline Модель & Четкая & Нечеткая \\
\hline Линейная & 3,34 & 3,30 \\
\hline Квадратичная & 2,94 & 2,82 \\
\hline
\end{tabular}

После идентификации на контрольной выборке данных выполнена проверка результатов моделирования для четких и нечетких окрестностных моделей с помощью относительных ошибок моделирования, вычисляемых по формуле (5) и представленных в табл. 2.

Таблица 2

Относительная ошибка моделирования, \%

\begin{tabular}{|c|c|c|}
\hline Модель & Четкая & Нечеткая \\
\hline Линейная & 4,69 & 4,65 \\
\hline Квадратичная & 4,30 & 4,22 \\
\hline
\end{tabular}

На рис. 2 и рис. 3 показаны относительные ошибки моделирования для фрагмента контрольной выборки, состоящего из 25 данных, для четких неиерархических и нечетких иерархических окрестностных моделей соответственно.

Проведя сравнение исходных и модельных данных для разработанных моделей процесса производства холоднокатаной стали, можно сделать вывод, что квадратичная окрестностная модель дает меньшую относительную ошибку по сравнению с линейной. Следует отметить, что введение нечеткой иерархической структуры приводит к несущественному снижению ошибок идентификации и моделирования, поэтому для практического применения можно рекомендовать четкие окрестностные модели рассматриваемого процесса.

Проанализировав на производственных данных результаты, полученные с помощью разработанных моделей, специалисты металлургического производства дали заключение о работоспособности предлагаемых методов и пригодности к применению для прогнозирования механических свойств металла, формирующихся в процессе непрерывного отжига. Разработанные математические модели признаны представляющими интерес для практического использования и рекомендованы для дальнейшего развития с учетом конкретных особенностей производства.

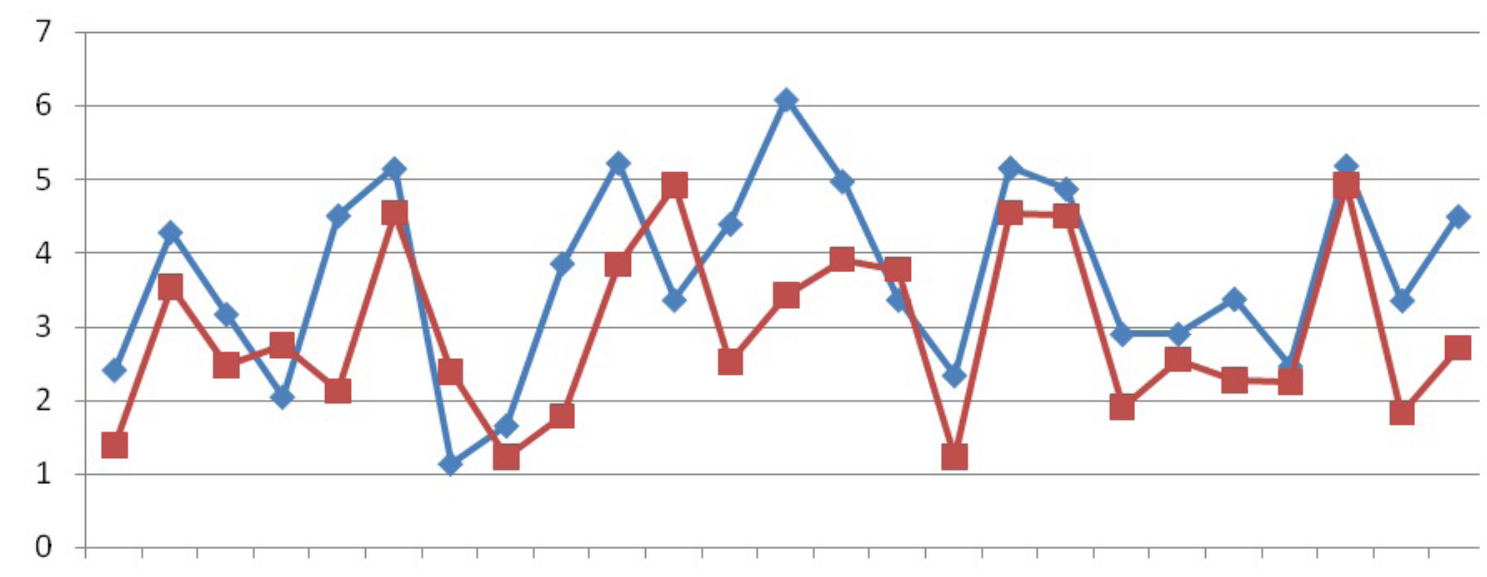

$\begin{array}{lllllllllllllllllllllllll}1 & 2 & 3 & 4 & 5 & 6 & 7 & 8 & 9 & 10 & 11 & 12 & 13 & 14 & 15 & 16 & 17 & 18 & 19 & 20 & 21 & 22 & 23 & 24 & 25\end{array}$

$\longrightarrow$ Линейная четкая $\quad-$ Квадратичная четкая

Рис. 2. Относительные ошибки моделирования для четких неиерархических окрестностных моделей 


\section{И. А. Сedblx}

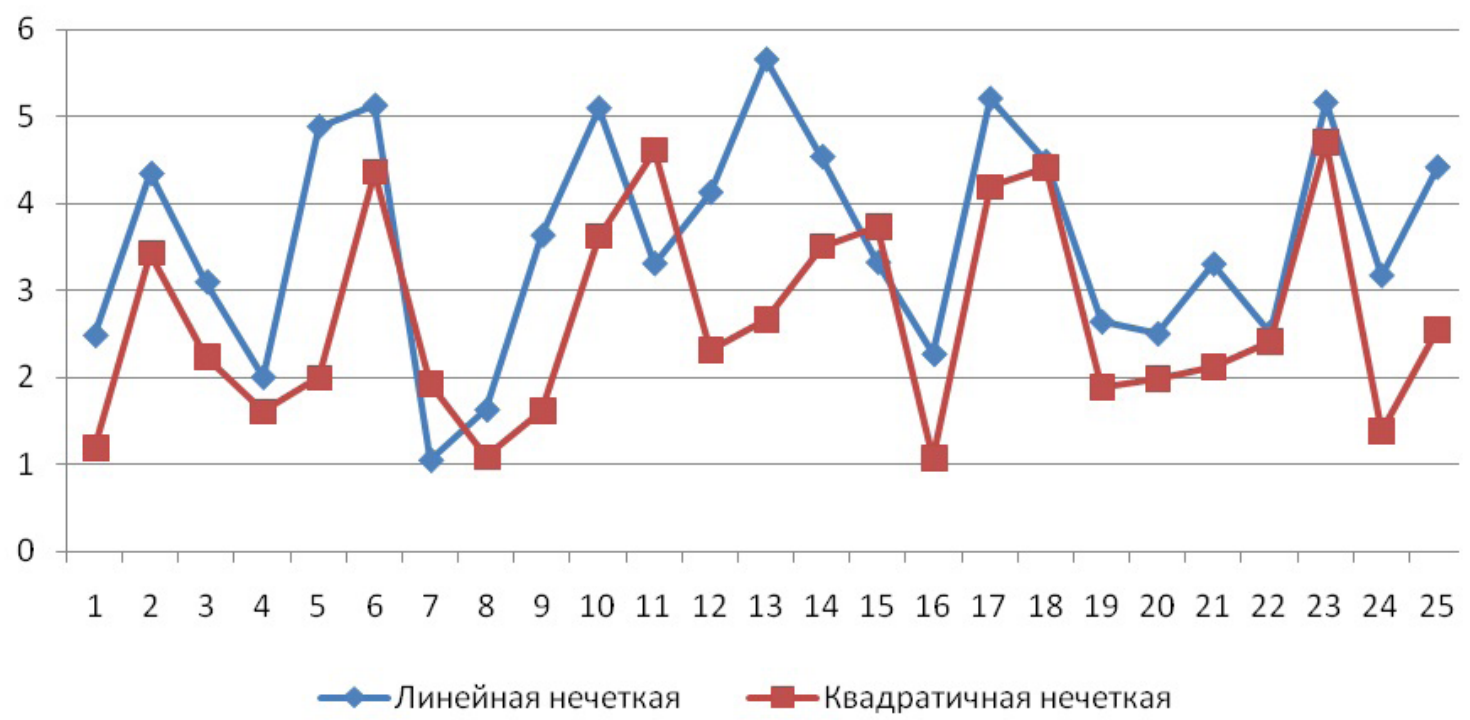

Рис. 3. Относительные очибки моделирования для нечетких иерархических моделей

\section{ЗАКЛЮЧЕНИЕ}

Таким образом, в работе рассмотрены основные механические свойства металла: предел текучести, временное сопротивление, относительное удлинение, определяемые при производстве холоднокатаной стали, для моделирования которого могут применяться динамические окрестностные модели.

Приведено определение динамической окрестностной модели с нечеткой иерархической структурой. Введено понятие двухуровневой нечеткой структуры, состоящей, в частности, из множества узлов первого и второго уровня, а также нечетких иерархических окрестностных связей между ними. Как частный случай рассмотрены линейные и квадратичные нечеткие иерархические окрестностные модели.

Построены линейные и квадратичные четкие и нечеткие окрестностные модели производства холоднокатаной стали в процессе непрерывного отжига, проведено сравнение результатов моделирования с производственными данными. В результате сравнения можно сделать вывод, что полученные для рассматриваемого процесса окрестностные модели с нечеткой иерархической структурой дают несущественное снижение относительных ошибок идентификации и моделирования по сравнению с неиерархическими моделями. Следовательно, для практическо- го применения рекомендуется использовать четкие окрестностные модели процесса производства стали.

\section{СПИСОК ЛИТЕРАТУРЫ}

1. Бобылев, А. В. Механические и технологические свойства металлов. Справочник / А. В. Бобылев - Москва : Металлургия, 1980. $296 \mathrm{c}$.

2.Золоторевскиц̆, В. С. Механические свойства металлов / В. С. Золоторевский. Москва: Металлургия, 1983. -350 с.

3. Гущина, М. С. Исследование влияния химического состава на механические свойства холоднокатаных полос из стали марки 08Ю / М. С. Гущина // «Технические науки - от теории к практике»: материалы XXIII международной заочной научно-практической конференции. (10 июля 2013 г.). - Новосибирск : Изд. «СибАК», 2013. - С. 59-68.

4. Гордеев, Е. Исследование механических свойств металла. Модель управления свойствами. - Режим доступа: // http://metallopraktik.ru/novosti/issledovaniemehanicheskih-svoystv-metalla-modelupravleniya/. - (Дата обращения: 29.12.2018).

5. Шмырин, А. М. Трилинейная окрестностная модель процесса формирования температуры смотки горячекатаной полосы / А. М. Шмырин, А. Г. Ярцев, В. В. Правильникова // Вестник ТГУ. Серия: Естественные 
и технические науки. - 2016. - Т. 21, вып. 2. C. $463-469$.

6. Shang, Y. Multi-agent coordination in directed moving neighborhood random networks / Y. Shang // Chinese Physics B. - 2010. - Vol. 19, №. 7. - Article ID 070201.

7. Parametrical neighborhood modelling of the process of forming the temperature of hotrolled strip coiling / A. M. Shmyrin [and others] // Journal of Chemical Technology and Metallurgy. - 2016. - 51(4). - C. 401-404.

8. Study of The Trilinear Neighborhood Model of Process of Formation of Temperature's Coiling Hot-Rolled Strip / A. M. Shmyrin [and others]// International Journal of Electrical and Computer Engineering (IJECE). - 2016. - Vol. 6, №. 3. - P. 1371-1374.

9. Блюмин, С. Л. Окрестностные системы / С. Л. Блюмин, А. М. Шмырин. - Липецк : ЛЭГИ, 2005. - 132 с.

10. Блюмин, С. Л. Билинейные окрестностные системы / С. Л. Блюмин, А. М. Шмырин, О. А. Шмырина. - Липецк: ЛЭГИ, 2006. - 131 с.

11. Седых, И. А. Окрестностное моделирование предела текучести стали после непрерывного отжига / И. А. Седых // Виртуальное моделирование, прототипирование и про-

Седых И. А. - канд. физ.-мат. наук, доцент кафедры высшей математики ФГБОУ ВО «Липецкий государственный технический университет».

E-mail: sedykh-irina@yandex.ru мышленный дизайн: материалы IV Международной научно-практической конференции (15-17 ноября 2017 г.). В 3 т. Т. 1.- Тамбов : Изд-во ТГТУ, 2017. - С. 378-383.

12. Седых, И. А. Управление динамическими окрестностными моделями с переменными окрестностями / И. А. Седых // Системы управления и информационные технологии. - 2018. - №1(71). - С. 18-23.

13. Седых, И. А. Двухуровневые полиномиальные динамические окрестностные модели с переменными окрестностями и их параметрическая идентификация / И. А. Седых // Вести высших учебных заведений Черноземья. - 2018. - №1(51). - С. 57-65.

14. $L i, Y$. Clustering coefficients of large networks. / Y. Li, Y. Shang, Y. Yang // Information Sciences. - 2017. - Vol. 382-383. - P. 350-358.

15. Scaled cluster consensus of discrete-time multi-agent systems with general directed topologies / B. Hou [and others] // Int. J. Control. 2016. - № 47. - P. 3839-3845.

Работа проведена в рамках выполнения государственного задания Министерства образования и науки России по проекту № 11.1446.2017/4.6.

Sedykh I. A. - candidate of Physics and Mathematics, Associate Professor of the Mathematics Department at the Lipetsk State Technical University.

E-mail: sedykh-irina@yandex.ru 